\title{
Lesion characteristics of acute myocardial infarction: an investigation with intravascular ultrasound
}

D Fukuda, T Kawarabayashi, A Tanaka, Y Nishibori, H Taguchi, Y Nishida, K Shimada, J Yoshikawa

\begin{abstract}
Objective-To use intravascular ultrasound (IVUS) to compare plaque morphology in acute myocardial infarction and stable angina pectoris.

Design-Retrospective study.

Setting-Primary care hospital.

Patients-59 consecutive cases of acute myocardial infarction and 50 consecutive cases of stable angina pectoris.

Methods-IVUS was used before coronary intervention.

Main outcome measures-Plaque morphology (incidence of eccentric plaque, subtle dissections, low echoic thrombus, calcification, echolucent areas, and bright speckled echo material), assessed visually using IVUS.

Results-There were no significant differences in plaque eccentricity or calcification between the two groups, but low echoic thrombus (acute myocardial infarction $15 \% v$ stable angina pectoris $0 \%$ ), subtle dissections ( $37 \% v 4 \%$ ), echolucent areas $(31 \% v 0 \%)$, and bright speckled echo material $(90 \% v 0 \%)$ were more common in the infarction group than in the stable angina group $(\mathrm{p}<0.001$ for all). There was a longer time between the onset of symptoms and the IVUS examination in patients with low echoic thrombus than in those without $(\mathrm{p}<0.03)$.

Conclusions-Low echoic thrombus, subtle dissections, echolucent areas, and bright speckled echo material are morphological characteristics associated with plaque at the time of acute myocardial infarction. These findings correspond pathologically to ruptured plaque.

(Heart 2001;85:402-406)
\end{abstract}

Keywords: intravascular ultrasound; acute myocardial infarction; plaque morphology

Intravascular ultrasound (IVUS) is an imaging technique that is capable of providing transluminal tomographic images of coronary arteries in vivo. $^{1-3}$ Although coronary angiography provides only a silhouette of the coronary artery lumen, the images that IVUS offers show the coronary artery wall, plaque morphology, and plaque composition. ${ }^{2-6}$ Thus the information about the coronary artery that IVUS provides can be of great use in determining coronary intervention strategies. ${ }^{17-11}$ As IVUS catheter technology has improved, the range and number of patients whom IVUS can benefit has increased considerably. ${ }^{3}$ It has further been reported that the negative contrast technique ${ }^{12}$ and coronary injection of normal saline are useful for enhancing the diagnostic capabilities of IVUS. While the investigation and assessment of the pathological characteristics of acute coronary syndromes is quite advanced, their mechanisms are still not well understood..$^{13}$ The high incidence of thrombus in culprit lesions ${ }^{14-16}$ in the acute phase of myocardial infarction is perhaps one reason why there has been insufficient investigation of plaque in these patients. The purpose of this study was to investigate plaque morphology in vivo at the time of acute myocardial infarction, and compare it with plaque morphology in stable angina pectoris.

\section{Methods}

PATIENTS

We studied 59 patients with acute myocardial infarction. All patients underwent coronary angiography and IVUS before intervention. For the purpose of this study, acute myocardial infarction was defined as follows: typical chest pain of more than 30 minutes' duration; creatine kinase > 150 IU; ECG evidence of acute myocardial infarction (ST elevation $>1 \mathrm{~mm}$ in contiguous leads or subendocardial pattern); and no contraindication to coronary angiography. We selected 50 target lesions in 50 patients with stable angina pectoris for comparison. Patients were included in this group if they satisfied the following requirements: complaint of angina on effort; ECG evidence of angina (ST depression $>2 \mathrm{~mm}$ during chest pain); and no contraindication to coronary angiography.

No patient in either group received any thrombolytic treatment. Risk factors such as hypercholesterolaemia (plasma cholesterol $\geqslant 220 \mathrm{mg} / \mathrm{dl}(5.68 \mathrm{mmol} / \mathrm{l})$ ), obesity (body mass index $\geqslant 25 \mathrm{~kg} / \mathrm{m}^{2}$ ), and a family history of ischemic heart disease were reviewed. During catheterisation, all patients received an initial bolus injection of $3000 \mathrm{IU}$ heparin and an additional $2000 \mathrm{IU}$ heparin by injection every hour. A 12 lead surface ECG was monitored continuously throughout the procedure. 


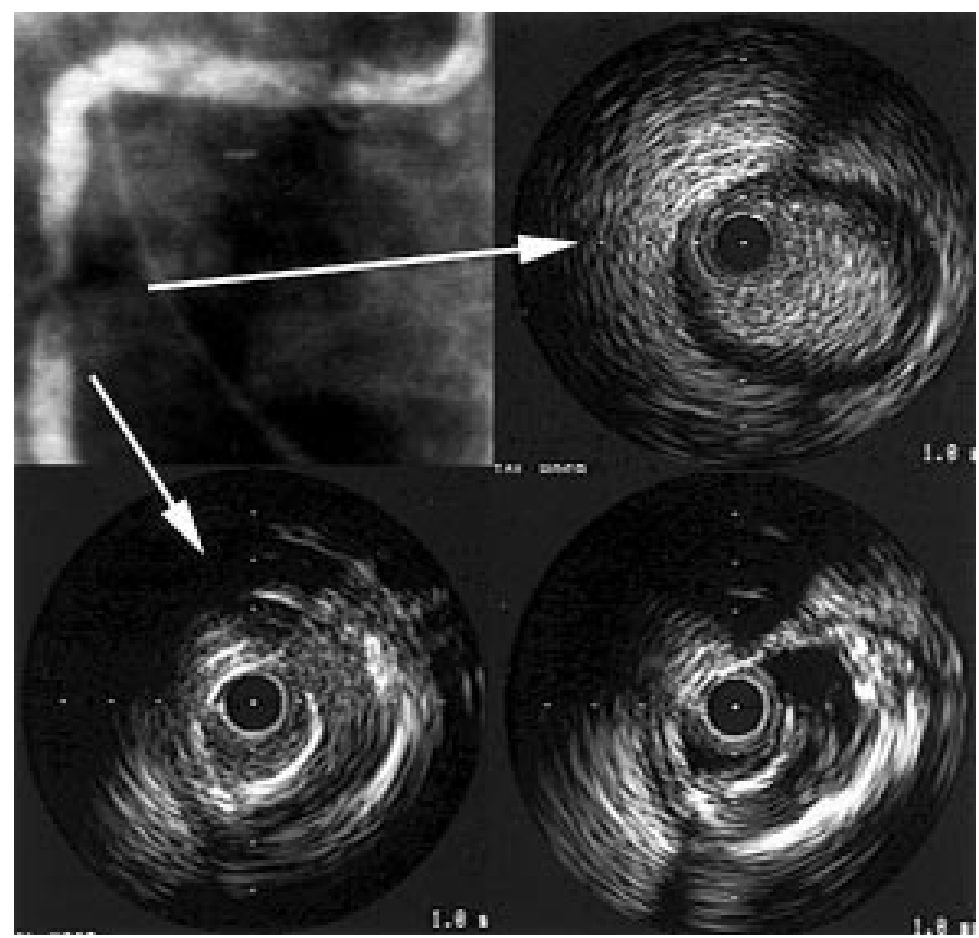

Figure 1 Coronary angiography and intravascular ultrasound (IVUS) images-1. The coronary angiography and IVUS images of a patient with acute myocardial infarction in the right coronary artery territory. ( $A$, top left) Coronary angiography shows the right coronary artery with $99 \%$ stenosis in the mid-portion. $(B$, top right) $A$ plain IVUS image of proximal stenosis. This shows bright speckled echo material. (C, bottom left) A plain IVUS image with suspected plaque dissection. (D, bottom right) Negative contrast image of $(C)$. This reveals the plaque dissection at 3 o'clock more clearly than the plain image.

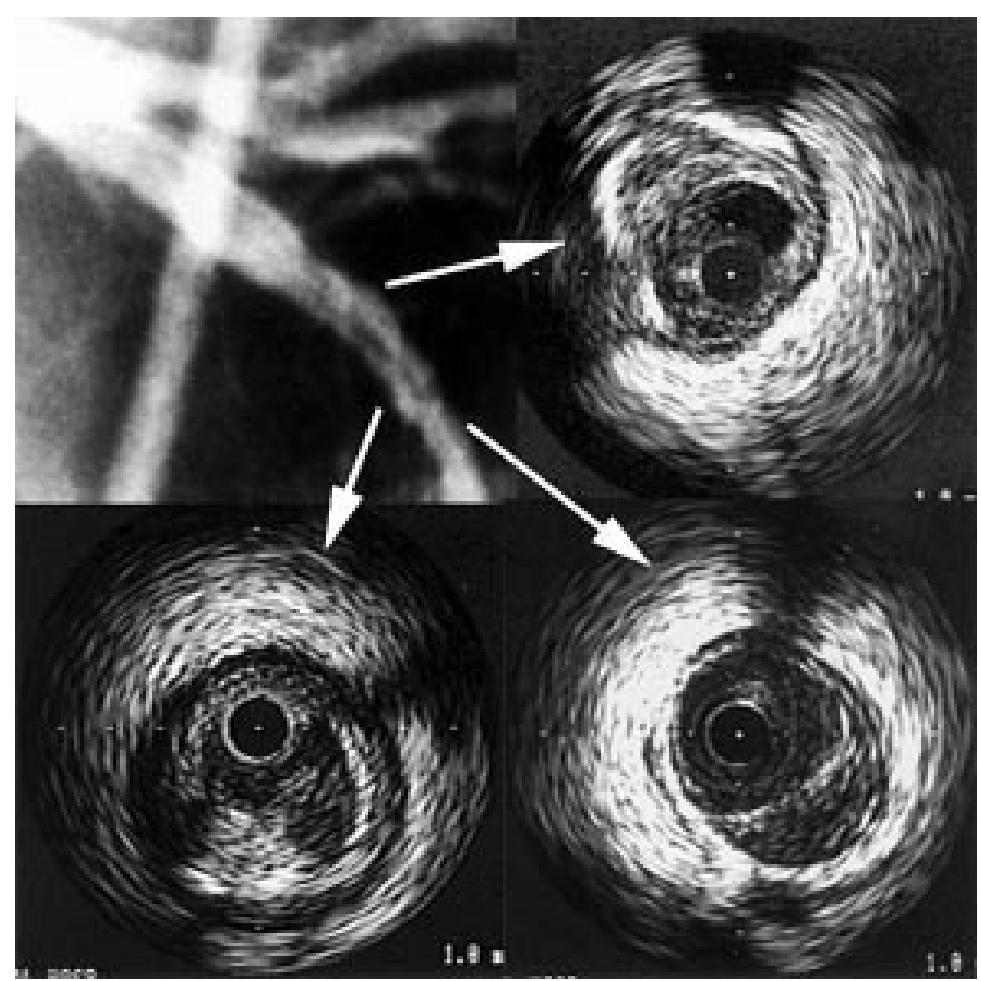

Figure 2 Coronary angiography and intravascular ultrasound (IVUS) images-2. The coronary angiography and IVUS images of a patient with acute myocardial infarction in left anterior descending coronary artery (LAD) territory. $(A$, top left) Coronary angiography shows filling defect in the mid-portion of the LAD. (B, top right) IVUS image of low echoic thrombus. In concordance with the angiographic findings, a low echoic mass representing thrombus can be seen at 12 to 2 o'clock. (C, bottom left) IVUS image with subtle dissection at 6 o'clock. (D, bottom right) IVUS image representing echolucent area at 4 to 6 o'clock; a low echoic lesion can be seen in the eccentric plaque.
The study protocol was approved by the ethics committee of Baba Memorial Hospital. We obtained written informed consent from all participants before coronary angiography.

IVUS IMAGING

IVUS imaging was performed using the UltraCross 3.2, $30 \mathrm{MHz}$ coronary imaging catheter (Boston Scientific, Boston, Massachusetts, USA) after intracoronary injection of $0.2 \mathrm{mg}$ glyceryl trinitrate. The IVUS catheter was advanced over a 0.014 inch $(0.36 \mathrm{~mm})$ guidewire to a point distal to the target lesion, the video recorder turned on, and an automatic pullback imaging run conducted at $0.5 \mathrm{~mm} / \mathrm{s}$ from distal to the target lesion to the guiding catheter. IVUS evaluation was performed in each case following coronary angiography and before intervention. If necessary, the procedure was repeated with a normal saline injection directly into the coronary artery as a negative contrast agent to wash blood from the lumen and prevent speckled reflections from red blood cells. ${ }^{12}$ Studies were recorded on high resolution s-VHS tape for off-line inspection and evaluation.

IVUS ANALYSIS

Quantitative measurements were obtained offline from the videotape. The cross sectional area of the external elastic membrane (EEM) was measured by tracing the leading edge of the adventitia. The target lesion site was taken to be the cross sectional slice with the smallest lumen. We also investigated arterial remodelling. Adaptive remodelling was defined as the target lesion showing more than a 5\% increase in EEM cross sectional area over the average reference area; constrictive remodelling was defined as the target lesion showing more than a $5 \%$ decrease in EEM area over average reference area.

Plaque morphology was assessed visually by two observers blinded to the results of coronary angiography. Plaque eccentricity and calcium deposition were assessed from the videotape, as was the incidence of bright speckled echo material (fig 1B), low echoic thrombus (fig 2B), subtle dissections (fig 2C), and echolucent areas (fig 2D), which are considered to be indicators of plaque rupture. Bright speckled echo material is already recognised to be one form of blood clot. ${ }^{17}{ }^{18}$ In this study, however, thrombus was defined as a low echoic mass, often mobile and extruding into the vessel lumen over several centimetres, and sometimes becoming detached from the vessel wall. We regarded low echoic thrombus and bright speckled echo material as separate entities. To investigate the differences between these IVUS blood clot images, patients with acute myocardial infarction were divided into two further subgroupsthose with and those without low echoic thrombus.

STATISTICAL ANALYSIS

Statistical analysis was performed using StatView J-5.0 (Abacus Concepts). Continuous data were compared using unpaired Student's $t$ tests. Categorical data were 
Table 1 Characteristics of patients and culprit lesions

\begin{tabular}{llll}
\hline & $\begin{array}{l}\text { Acute myocardial } \\
\text { infarction }\end{array}$ & Stable angina & p Value \\
\hline $\begin{array}{l}\text { Patient characteristics } \\
\mathrm{n}\end{array}$ & 59 & 50 & \\
Male & $43(73 \%)$ & $38(76 \%)$ & $\mathrm{NS}$ \\
Age (years) (mean (SD)) & $62.5(9.8)$ & $62.8(8.7)$ & $\mathrm{NS}$ \\
Coronary risk factors & & & \\
Diabetes mellitus & $24(41 \%)$ & $9(18 \%)$ & 0.06 \\
Hypertension & $38(64 \%)$ & $18(36 \%)$ & $\mathrm{NS}$ \\
Hypercholesterolaemia & $28(47 \%)$ & $18(36 \%)$ & $\mathrm{NS}$ \\
Family history & $2(3 \%)$ & $3(6 \%)$ & $\mathrm{NS}$ \\
Obesity & $15(25 \%)$ & $11(22 \%)$ & $\mathrm{NS}$ \\
Smoking & $44(75 \%)$ & $20(40 \%)$ & 0.06 \\
& & & \\
Culprit lesion & $33(56 \%)$ & $27(54 \%)$ & $\mathrm{NS}$ \\
LAD & $4(7 \%)$ & $6(12 \%)$ & $\mathrm{NS}$ \\
LCx & $22(37 \%)$ & $17(34 \%)$ & NS \\
RCA & & &
\end{tabular}

LAD, left anterior descending coronary artery; LCx, left circumflex coronary artery; RCA, right coronary artery.

Table 2 Findings on intravascular ultrasound: vessel size

\begin{tabular}{llll}
\hline & $\begin{array}{c}\text { Acute myocardial } \\
\text { infarction }(n=59)\end{array}$ & $\begin{array}{l}\text { Stable angina } \\
(n=50)\end{array}$ & $p$ Value \\
\hline $\begin{array}{llll}\text { Distal site } \\
\text { EEM CSA }\left(\mathrm{mm}^{2}\right)\end{array}$ & $13.4(4.5)$ & $13.4(3.4)$ & $\mathrm{NS}$ \\
Lumen CSA $\left(\mathrm{mm}^{2}\right)$ & $7.10(3.3)$ & $7.90(1.4)$ & $\mathrm{NS}$ \\
$\begin{array}{l}\text { Proximal site } \\
\text { EEM CSA }\left(\mathrm{mm}^{2}\right)\end{array}$ & $16.2(5.4)$ & $16.3(3.7)$ & $\mathrm{NS}$ \\
Lumen CSA $\left(\mathrm{mm}^{2}\right)$ & $8.5(3.4)$ & $9.2(2.5)$ & $\mathrm{NS}$ \\
Target site & $13.9(4.6)$ & $12.8(3.0)$ & $\mathrm{NS}$ \\
EEM CSA $\left(\mathrm{mm}^{2}\right)$ & & &
\end{tabular}

Values are mean (SD).

CSA, cross sectional area; EEM, external elastic membrane.

Table 3 Findings on intravascular ultrasound:plaque morphology

\begin{tabular}{llll}
\hline & $\begin{array}{l}\text { Acute myocardial } \\
\text { infarction }(n=59)\end{array}$ & $\begin{array}{l}\text { Stable angina } \\
(n=50)\end{array}$ & $p$ Value \\
\hline $\begin{array}{l}\text { Eccentric plaque } \\
\text { Calcification }\end{array} \quad 30(51 \%)$ & $24(48 \%)$ & $\mathrm{NS}$ \\
$\quad$ Superficial & $24(41 \%)$ & $12(24 \%)$ & $\mathrm{NS}$ \\
$\quad \begin{array}{l}\text { Deep } \\
\text { Subtle dissection }\end{array}$ & $22(37 \%)$ & $18(36 \%)$ & $\mathrm{NS}$ \\
Echolucent area & $22(37 \%)$ & $2(4 \%)$ & $<0.001$ \\
Low echoic thrombus & $13(31 \%)$ & 0 & $<0.001$ \\
Bright speckled echo material & $9(15 \%)$ & 0 & $<0.001$ \\
\hline
\end{tabular}

compared using $\chi^{2}$ analysis of Fisher's exact test. A probability value $\mathrm{p}<0.05$ was considered significant.

\section{Results}

PATIENT CHARACTERISTICS

Patient characteristics and lesion characteristics are shown in table 1 . There was no significant difference in sex or age between the two groups. There were also no significant differences in coronary risk factors between the groups, although there was a trend for diabetes mellitus and smoking to be more prevalent in the group with acute myocardial infarction.

\section{LESION CHARACTERISTICS}

Lesion location (target vessel) was statistically similar between the two groups. In half the cases, the culprit lesion was found in the left anterior descending coronary artery (table 1). The results of the quantitative measurements of vessel size are shown in table 2. There were no differences in the EEM cross sectional area of the culprit lesion between the two groups (mean (SD)): infarct patients $13.9(4.6) \mathrm{mm}^{2} v$

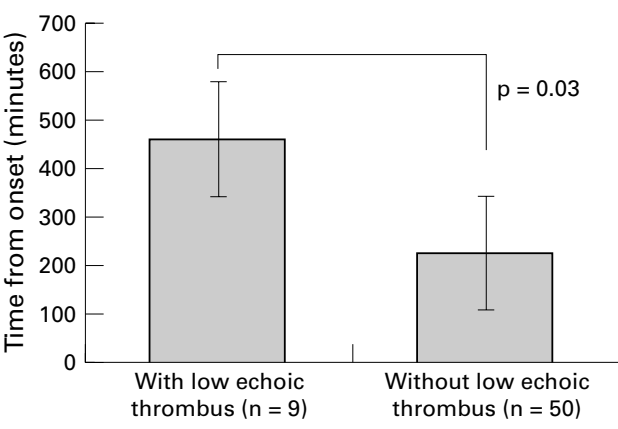

Figure 3 Comparison of time from onset of symptoms between cases with and without low echoic thrombus. The time course in cases with low echoic thrombus was significantly longer than in those without low echoic thrombus.

stable angina $12.8(3.0) \mathrm{mm}^{2}$; NS. Adaptive arterial remodelling was seen more often in the infarct group than in stable angina group: infarct patients $31 \% \quad v$ stable angina $14 \%$; $\mathrm{p}<0.05$. There was no significant difference in constrictive remodelling between the groups.

\section{PLAQUE MORPHOLOGY}

We were able to use IVUS and negative contrast injection in all patients in the study and there were no major complications. Thus we were able to obtain information about plaque morphology which could not have been acquired by coronary angiography alone (fig 1A, fig 2A). Plaque morphology was assessed for the incidence of subtle dissections, echolucent areas, low echoic thrombus, and bright speckled echo material, as shown in table 3. These characteristics were more clearly observable with negative contrast injection (fig 1C, D). Plaque eccentricity and calcification were similar across the groups. In the infarct group, however, plaque was more often characterised by bright speckled echo material (fig 1B), low echoic thrombus (fig 2B), subtle dissections (fig 2C), and echolucent areas (fig 2D) than in the stable angina group.

ELAPSED TIME FROM ONSET AND TYPE OF THROMBUS

In the infarct subgroup with low echoic thrombus, the time from the onset of symptoms was longer than in the subgroup without low echoic thrombus (fig 3). However, there were no other significant differences between cases with and without low echoic thrombus.

\section{Discussion}

IVUS WITH NEGATIVE CONTRAST

IVUS provides transluminal tomographic images of coronary arteries in vivo, allowing visual assessment of the atherosclerotic disease process. ${ }^{6}{ }^{19}$ Previous studies have described plaque morphology in patients with unstable and stable angina and the characteristics of unstable plaques. ${ }^{213}{ }^{20-22}$ However, there have been few investigations of plaque in lesions associated with acute myocardial infarction. The reason for this has been the high incidence of thrombus in culprit lesions in the acute phase of myocardial infarction, ${ }^{14}$ causing the image of the plaque to be obscured. In this study we 
used IVUS and negative contrast injection of normal saline solution directly into the coronary arteries to investigate the characteristics of plaque in the lesions of acute myocardial infarction. Subtle dissections and echolucent areas were observed in $37 \%$ and $31 \%$ of cases, respectively. Although patients were selected for this study, these percentages were higher than previously reported. ${ }^{15}$ We believe that negative contrast injection contributed to these results.

CHARACTERISTICS OF PLAQUE IN ACUTE MYOCARDIAL INFARCTION

Pathology reports have shown that plaque rupture and thrombus formation cause acute myocardial infarction. ${ }^{14}$ An echolucent area within the plaque signifies a ruptured plaque, with a thin membrane representing the fibrous cap or deep ulceration in the plaque. ${ }^{20}$ The subtle dissections present in plaque are said to represent intimal dissection of the coronary artery. Our study showed that subtle dissections, echolucent areas, low echoic thrombus, and bright speckled echo material are significantly more common in acute myocardial infarction than in stable angina. These results are in line with previous reports ${ }^{15}$ and correspond to the pathological findings. In the infarct group, however, there were some cases without the characteristics of plaque rupture. Although plaque rupture is thought to be one of the main causal mechanisms of acute myocardial infarction, it has been reported that plaque erosion also causes thrombus formation, and if such thrombus occludes the lumen, acute infarction may occur. ${ }^{23}{ }^{24} \mathrm{We}$ suppose that plaque erosion may be one reason why some cases did not have the characteristics of ruptured plaque on IVUS.

IVUS imaging showed that vessels containing the culprit lesions were similar in size in acute myocardial infarction and stable angina. These results correspond to previous pathological findings suggesting that plaque rupture depends more on plaque type than on vessel size. $^{202526}$ However, patients in the infarct group more often showed adaptive remodelling than patients in the stable angina group. There have been many recent reports on arterial remodelling and our results are in agreement with their findings. Atherosclerosis is considered to be an important reason for arterial remodelling; however, there are many definitions of this process, and further investigation is needed. ${ }^{82}$

We observed two patients in the stable angina group with subtle dissections. These did not differ in size or extent from those seen in the infarct group, but there were no clear echolucent areas in the angina patients. This suggests that not all cases of plaque rupture cause acute myocardial infarction, and also that both plaque rupture itself and also the contents of the rupturing plaque-notably thrombogenic agents represented by an echolucent area-play an important role in initiating the onset of acute myocardial infarction.

In a previous study it was reported that the degree of calcification of a plaque is directly related to its stability. ${ }^{20}$ In our study, no signifi- cant difference was observed between the two groups in terms of plaque calcification, although superficial calcification tended to be more common in the infarct group. Calcium deposition on the plaque surface is thought to be a cause of plaque instability. ${ }^{21}$ It is already known that plaque rupture and healing play an important role in the processes of atherosclerotic progression and regression. ${ }^{20}$

THROMBUS FORMATION AT THE LESION

In culprit lesions in acute myocardial infarction, we often observed low echoic thrombus and bright speckled echo material. We suspect that time from symptom onset may be an important factor in the differences between these two types of blood clot. With a few exceptions, we saw a high incidence of low echoic thrombus and bright speckled echo material in culprit lesions from the infarct patients, and we concluded that these features are characteristic of acute myocardial infarction. This would also correspond to the pathological findings. ${ }^{14}$ Our results suggest that the plaque characteristics of acute myocardial infarction revealed by IVUS may change greatly over time. It is therefore necessary to take the time course into consideration when assessing the characteristics of plaques responsible for acute myocardial infarction.

\section{CONCLUSIONS}

The low echoic thrombus, subtle dissections, echolucent areas, and bright speckled echo material were common occurrences in plaque in target vessels in the acute phase of myocardial infarction. IVUS was found to be a useful tool for investigating plaque characteristics, especially when combined with negative contrast. Our results are in agreement with the pathological finding that plaque rupture and subsequent thrombus formation causes the onset of acute myocardial infarction.

1 Bruchhauser J, Sechtem U, Hopp HW, et al. Intracoronary ultrasound changes the therapeutic approach in ambivalent angiography findings. $Z$ Kardiol 1997;86:138-47.

2 Alfonso F, Macaya C, Goicolea J, et al. Intravascular ultrasound imaging of angiographically normal coronary segments in patients with coronary artery disease. Am Heart F 1994;127:536-44.

3 Metz JA, Yock PG, Fitzgerald PJ. Intravascular ultrasound: basic interpretation. Cardiol Clin 1997;15:1-15.

4 De Scheerder I, De Man F, Herregods MC, et al. Intravascular ultrasound versus angiography for measurement of luminal diameters in normal and diseased coronary arteries. Am Heart $\mathcal{F}$ 1994;127:243-51.

5 Gorge G, Ge J, Haude M, et al. Intravascular ultrasound for evaluation of coronary arteries. Herz 1996;21:78-89.

6 Erbel R, Ge J, Gorge G, et al. Intravascular ultrasound classification of atherosclerotic lesions according to American Heart Association recommendation. Coronary Artery can Heart Association rec

7 Albiero R, Rau T, Schluter M, et al. Comparison of immediate and intermediate-term results of intravascular ultrasound versus angiography-guided Palmaz-Schatz stent implantation in matched lesions. Circulation 1997;96: 2997-3005.

8 Dangas G, Mintz GS, Mehran R, et al. Preintervention arterial remodeling as an independent predictor of target-lesion revascularization after nonstent coronary intervention: an analysis of 777 lesions with intravascular ultrasound imaging. Circulation 1999;99:3149-54.

9 Hoffmann R, Mintz GS, Mehran R, et al. Tissue proliferation within and surrounding Palmaz-Schatz stents is dependent on the aggressiveness of stent implantation technique. Am $\mathcal{F}$ Cardiol 1999;83:1170-4.

10 Abizaid AS, Mintz GS, Mehran R, et al. Long-term follow-up after percutaneous transluminal coronary angioplasty was not performed based on intravascular ultrasound findings: importance of lumen dimensions. Circulation 1999;100:256-61. 
11 Schroeder S, Baumbach A, Haase KK, et al. Reduction of restenosis by vessel size adapted percutaneous transluminal coronary angioplasty using intravascular ultrasound. $A m \mathcal{F}$

12 Honye J, Saito S, Takayama T, et al. Clinical utility of negative contrast intravascular ultrasound to evaluate plaque morphology before and after coronary interventions. Am $\mathcal{F}$ Cardiol 1999;83:687-90.

13 Namiki N, Uchiyama T, Nagai Y, et al. Graphical comparison of coronary arterial culprit lesions in acute myocardial infarction and unstable angina pectoris. Intern Med 1999;38:849-55.

14 Brosius FC, Roberts WC. Significance of coronary arterial thrombus in transmural acute myocardial infarction. A study of 54 necropsy patients Circulation 1981;63:810-16.

5 Bocksch W, Schartl M, Beckmann S, et al. Intravascular ultrasound imaging in patients with acute myocardial infarction. Eur Heart f 1995;16:46-52.

16 Bocksch W, Schartl M, Beckmann S, et al. Intravascular ultrasound assessment of direct percutaneous transluminal ultrasound assessment of direct percutaneous transluminal coronary angioplasty in patients with acute

17 Bhandari AK, Nanda NC, Hicks DG. Two-dimensional echocardiography of intracardiac masses: echo patternhistopathology correlation. Ultrasound Med Biol 1982;8 673-80.

18 Frimerman A, Miller HI, Hallman M, et al. Intravascular ultrasound characterization of thrombi of different composition. Am f Cardiol 1994;73:1053-7.
19 Ge J, Erbel R, Gerber T, et al. Intravascular ultrasound imaging of angiographically normal coronary arteries: a prospective study in vivo. Br Heart $\mathcal{F}$ 1994;71:572-8.

20 Ge J, Chirillo F, Schwedtmann J, et al. Screening of ruptured plaques in patients with coronary artery disease by intravascular ultrasound. Heart 1999;81:621-7.

21 Ge J, Baumgart D, Haude M, et al. Role of intravascular ultrasound imaging in identifying vulnerable plaques. Herz 1999;24:32-41.

22 Weissman NJ, Sheris SJ, Chari R, et al. Intravascular ultrasonic analysis of plaque characteristics associated with coronary artery remodeling. Am ₹ Cardiol 1999;84:37-40.

23 Van der Wal AC, Becker AE, van der Loos CM, et al. Site of intimal rupture or erosion of thrombothed coronary atherosclerotic plaque is characterized by an inflammatory process irrespective of the dominant plaque morphology. Circulation 1994;89:36-44

24 Kojima S, Nonogi H, Miyao Y, et al. Is preinfarction angina related to the presence or absence of coronary plaque rupture? Heart 2000;83:64-8.

25 Yamagishi $M$, Terashima M, Awano K, et al. Morphology of vulnerable coronary plaque: insights from follow-up of patients examined by intravascular ultrasound before an acute coronary syndrome. $7 \mathrm{Am}$ Coll Cardiol 2000;35:10611.

26 Yock PG. The future of minimally invasive myocardial revascularization: a cardiologist's view. $f$ Card Surg 1998;13:310-15.

\section{A combination of persistent left superior vena cava and a large secundum atrial septal defect in a 34 year old woman}

A 34 year old woman presented with increasing shortness of breath, and a secundum atrial septal defect (ASD) was diagnosed by transthoracic echocardiography. A preoperative transoesophageal echocardiogram demonstrated a large secundum ASD with left to right shunt, and a dilated right ventricle (RV) with a pulmonary artery systolic pressure of $60 \mathrm{~mm} \mathrm{Hg}$ (below left, * indicates ASD). The coronary sinus (CS) was noted to be dilated, and an injection of contrast into the left antecubital vein resulted in opacification of the CS and then the right atrium (RA) (below right). Injection into the right antecubital vein was then performed leading to the normal sequence of opacification. All pulmonary veins were shown to drain normally into the left atrium (LA). The surgical findings were that of a $3 \times 2 \mathrm{~cm}$ secundum ASD and a large CS receiving the left superior vena

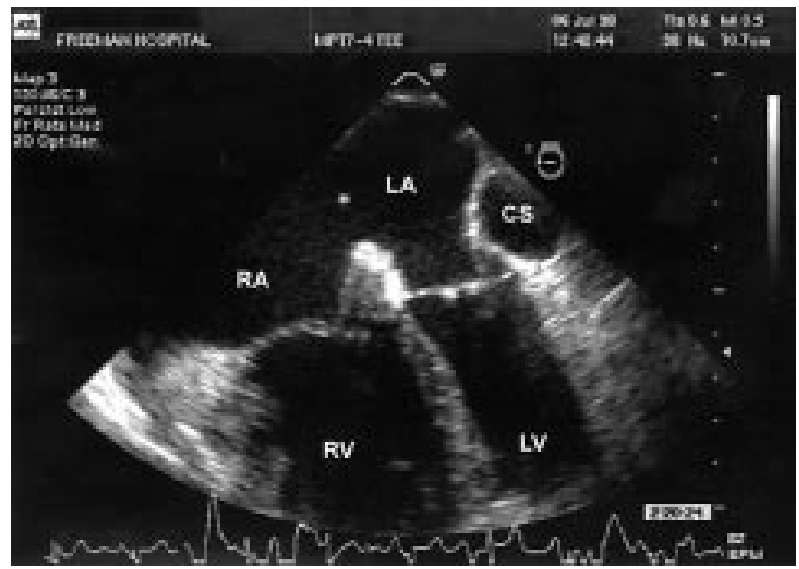

cava (LSVC). The LSVC was drained via a sucker in the CS. A persistent LSVC is the most common congenital anomaly involving the systemic veins. In most cases the LSVC drains into the RA by way of the CS, and the systemic venous return is physiologically normal. Preoperative identification of the anomaly is important, however, because the caval cannulation may need to be altered in order to drain the LSVC adequately during cardiopulmonary bypass. If a surgical procedure requiring an open RA is planned, the LSVC should be cannulated either directly or via the CS ostium. In addition the use of retrograde cardioplegia will be precluded.

THEODORA ZAGLAVARA J R L HAMILTON ANTOINETTE KENNY antoinette.kenny@ncl.ac.uk

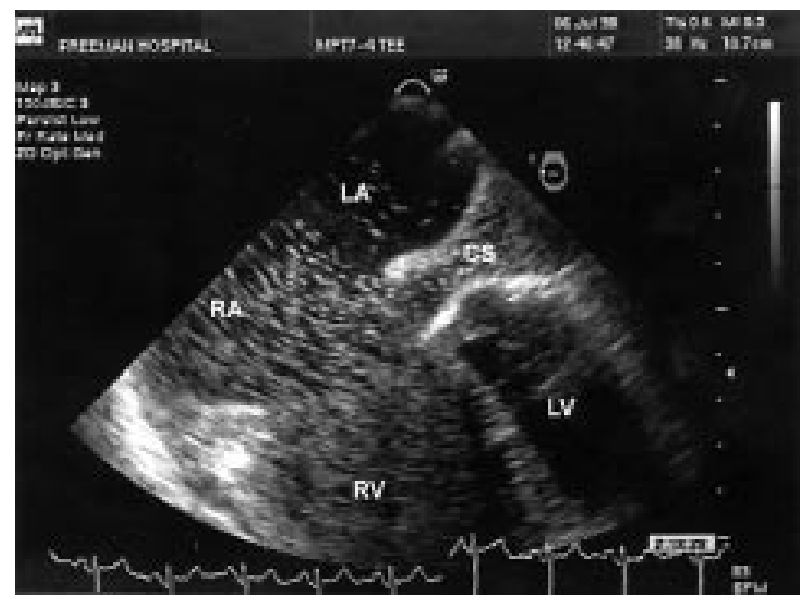

